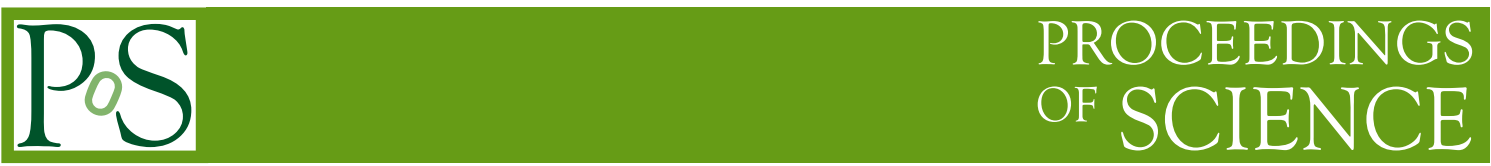

\title{
Recent lattice results on finite temperature and density QCD, part I
}

\author{
Frithjof Karsch ${ }^{* \dagger}$ \\ Physics Department, Brookhaven National Laboratory, Upton, NY 11973, USA \\ E-mail: karschebnl.gov
}

We discuss recent progress made studies of bulk thermodynamics of strongly interacting matter through lattice simulations of QCD with an almost physical light and strange quark mass spectrum. We present results on the QCD equation of state at vanishing and non-vanishing quark chemical potential and show first results on baryon number and strangeness fluctuations, which might be measured in event-by-event fluctuations in low energy runs at RHIC as well as at FAIR.

Critical Point and Onset of Deconfinement 4th International Workshop

July 9-132007

GSI Darmstadt,Germany

\footnotetext{
* Speaker.

${ }^{\dagger}$ This work has been supported in part by contract DE-AC02-98CH10886 with the U.S. Department of Energy.
} 


\section{Introduction}

The striking observations of large elliptic flow and jet quenching at RHIC [1] hints at an early equilibration of the dense matter formed in relativistic heavy ion collisions. This in turn suggests that the constituents of this medium, which at RHIC equilibrates at temperatures less than about $300 \mathrm{MeV}$ or energy densities of about $15 \mathrm{GeV} / \mathrm{fm}^{3}$, are still interacting strongly. The equation of state (EoS) describing this medium thus should exhibit significant deviations from ideal gas behavior. This has indeed been seen in calculations of the QCD equation of state on the lattice, where deviations from the non-interacting limiting behavior, $\varepsilon=3 p$, show up as a large peak in the trace anomaly in units of $T^{4}, \Theta^{\mu \mu}(T) / T^{4} \equiv(\varepsilon-3 p) / T^{4}$.

Pretty soon bulk thermodynamics, and in particular the EoS, will be probed at even higher temperatures at the LHC. This will be complemented by experimental studies of matter at lower temperature but non-zero baryon number density through low energy heavy ion collisions at RHIC and at the future European heavy ion facility, FAIR, at GSI/Darmstadt. It thus will be of importance to get control over the EoS in a wide range of temperatures and to analyze also its dependence on the baryon number density or the baryon chemical potential.

While good control over the continuum extrapolation of numerical results on the EoS has been reached in lattice calculations for the $\mathrm{SU}(3)$ gauge theory, much work is still needed to get similarly good control over calculations performed with physical light and strange quark masses. We will discuss here the current status of these lattice calculations.

During recent years the steady progress made in lattice calculations through important new algorithmic developments [2] and increased computing resources helped to greatly advance our knowledge of the thermodynamics of strongly interacting elementary particles [3]. On the one hand, lattice calculations of the equation of state [4, 5, 6] and transition temperature [7, 8, , , with an almost realistic quark mass spectrum are now possible at vanishing chemical potential. On the other hand, lattice calculations based on various approximation schemes now also provide first insight into the thermodynamics at non-zero quark or baryon chemical potential $\left(\mu_{q}\right)$ [10].

In this review we will discuss some of the results obtained recently with $\mathscr{O}\left(a^{2}\right)$ improved staggered fermion formulations. As these results have been presented in July/August of 2007 in a very similar format at the '4th International Workshop on Critical Point and Onset of Deconfinement' and at the 'XXV International Symposium on Lattice Field Theory' the write-up of these talks has been splitted into two parts. In this first part we will focus on recent results on the equation of state at vanishing and non-vanishing chemical potential and discuss results on strangeness and baryon number fluctuations which may become detectable in heavy ion collisions. Part II [11] focuses on a discussion of results on the nature of the QCD transition and reports on the current status of calculations of the transition temperature in QCD.

\section{The QCD equation of state}

While continuum extrapolated results for the EoS of the SU(3) gauge theory have been established more than 10 years ago [3], this still has to be achieved for QCD with a physical quark mass spectrum. Lattice calculations with light dynamical quarks, of course, introduce their own set of problems into the numerical study of QCD thermodynamics. The well-known problems with the 

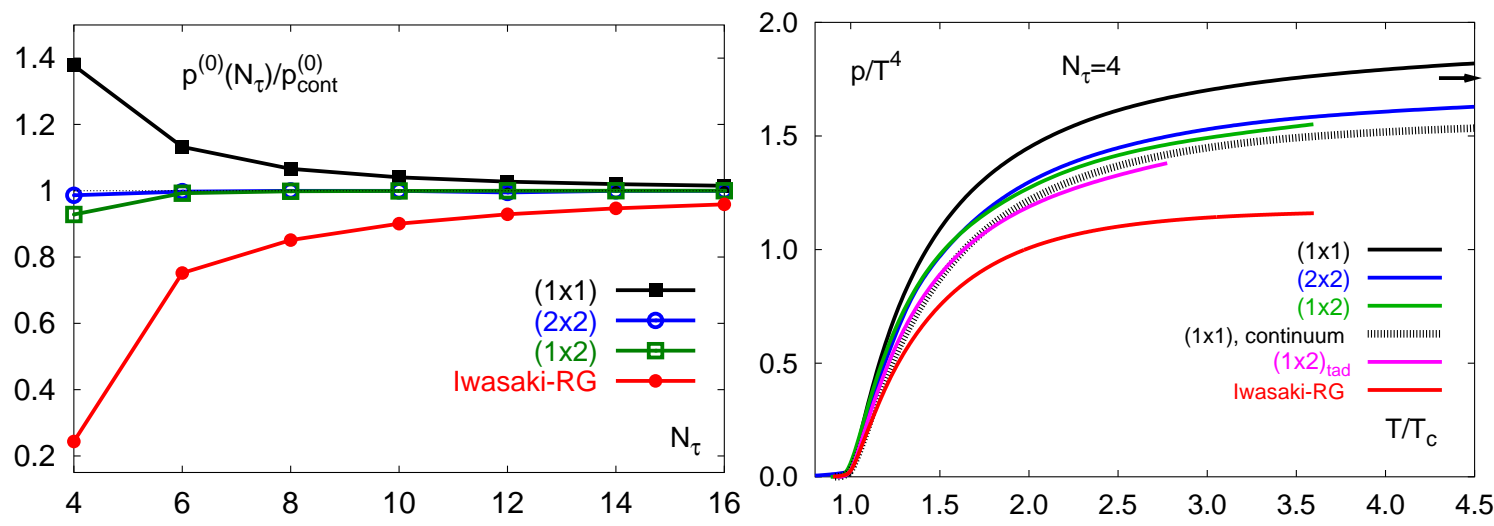

Figure 1: Pressure in the SU(3) gauge theory calculated for the standard Wilson action $(1 \times 1)$ and several improved actions analytically in the infinite temperature, ideal gas limit on lattices with temporal extent $N_{\tau}$ (left). The right hand figure shows numerical results obtained on lattices with temporal extent $N_{\tau}=4$.

discretization of fermion actions that require to break the chiral symmetry of the continuum action and/or requires the introduction of additional fermion species leads to a cut-off dependent modification of the hadron spectrum that will influence thermodynamic properties at low temperatures, i.e. in the hadronic phase. This introduces additional cut-off dependencies into lattice studies of QCD thermodynamics that are not present in pure gauge theories and require a detailed analysis. Aside from this, however, studies of QCD thermodynamics suffer from similar discretization errors as pure gauge theories which strongly influence studies of bulk thermodynamics in the plasma phase of QCD and, in particular, show up as a strong cut-off dependence of the high temperature limit of bulk thermodynamic observables. These cut-off effects essentially arise from the need to replace derivatives in the QCD Lagrangian by finite differences. In the next subsection we briefly recall the importance of these effects for the analysis of thermodynamics in the high temperature phase of SU(3) gauge theories before starting to discuss the thermodynamics of QCD with two degenerate, light quark masses and a heavier strange quark mass.

\subsection{Cut-off effects in QCD thermodynamics}

\subsubsection{SU(3) gauge theories revisited}

In the standard approach to the discretization of a SU(3) gauge theory the Euclidean action is represented by a sum over $(1 \times 1)$-Wilson loops (plaquettes). This introduces $\mathscr{O}\left(a^{2}\right)$ discretization errors. It is well known how to eliminate these leading order discretization errors on the tree level by adding larger Wilson loops, eg. $(1 \times 2)$ or $(2 \times 2)$ loops. This shifts the leading order discretization errors to $\mathscr{O}\left(a^{4}\right)$. Other improvement schemes, like for instance the Iwasaki action, improve certain non-perturbative features of the lattice action, but do not aim at an elimination of the $\mathscr{O}\left(a^{2}\right)$ errors. These discretization errors also show up in lattice studies of thermodynamic quantities on lattices with finite temporal extent $\left(N_{\tau}\right)$; as the relevant scale is the lattice cut-off in units of the temperature, $a T \equiv 1 / N_{\tau}, \mathscr{O}\left(a^{n}\right)$ cut-off errors thus show up in finite temperature calculations as $\mathscr{O}\left(1 / N_{\tau}^{n}\right)$ errors. This can explicitly been analyzed in the infinite temperature ideal gas limit of QCD. In Fig.1(1)ft) 


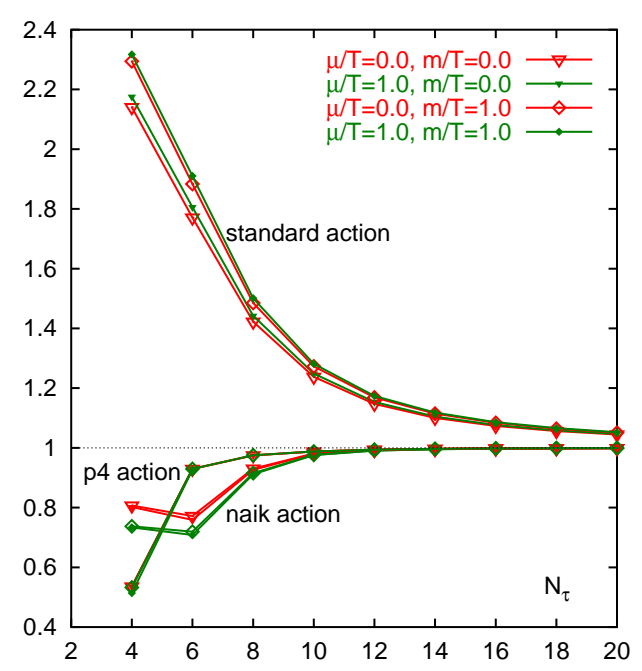

Figure 2: Cut-off dependence of the infinite temperature ideal gas pressure in staggered fermion formulations of QCD thermodynamics. Shown are analytic results for the pressure calculated in the thermodynamic limit $\left(N_{\sigma} \rightarrow \infty\right)$ on lattices with temporal extent $N_{\tau}$ for the standard 1-link action as well as two $\mathscr{O}\left(a^{2}\right)$ improved actions. Results are shown for vanishing and non-vanishing values of the chemical potential $(\mu / T=0,1)$.

we show the cut-off dependence of the pressure calculated analytically for various $\mathrm{SU}(3)$ actions as function of $N_{\tau}$ and normalized to the continuum $\left(N_{\tau} \rightarrow \infty\right)$ Stefan-Boltzmann value.

One lesson learned in studies of the EoS of a pure gauge theory is that it is extremely important to use at least $\mathscr{O}\left(a^{2}\right)$ improved gauge actions for the calculation of bulk thermodynamic observables like eg. energy density and pressure, if one wants to reduce cut-off effects at high temperature already on lattices with small temporal extent. As can be seen in Fig. 11(right) also at temperature close to the deconfinement transition temperature cut-off effects on lattices with fixed temporal extent $\left(N_{\tau}=4\right)$ follow the pattern seen already in the infinite temperature limit. While the pressure calculated with actions that have $\mathscr{O}\left(a^{2}\right)$ cut-off errors deviates strongly from the continuum extrapolated result also in the vicinity of the transition temperature calculations with $\mathscr{O}\left(a^{2}\right)$ improved gauge actions yield results on coarse latices that are already close to the continuum extrapolated result.

\subsubsection{Thermodynamics with staggered fermions}

These generic features also hold true in calculations performed in QCD with light quarks. The standard discretization scheme of the fermionic part of the QCD action (1-link actions) introduces $\mathscr{O}\left(a^{2}\right)$ errors that can be removed in thermodynamic observables on the tree level by introducing suitably chosen additional 3-link terms (Naik, p4). The resulting cut-off effects in the infinite temperature, ideal gas limit are shown in Fig. \&. We note that the improvement schemes improve bulk thermodynamic observables at vanishing as well as non-vanishing chemical potential. These actions are usually supplemented with additional terms that reduce the flavor symmetry breaking 
(smearing) and/or reduce cut-off effects beyond the tree level (tadpole improvement) ${ }^{1}$. None of these improvements, however, alter the behavior in the infinite temperature limit. They are thus not expected to be essential for studies of bulk thermodynamics in the high temperature phase of QCD.

The early calculations with unimproved staggered fermions [12] and calculations with unimproved Wilson fermions [13] as well as recent calculations with the 1-link, stout smeared action action [ $\llbracket$ indeed showed discretization errors similar to those observed in pure gauge theories; at high temperature energy density and pressure deviate strongly from the continuum ideal gas behavior and reflect the large cut-off dependence of the ideal quark-gluon gas in these discretization schemes $^{2}$. We therefore will focus in the following on a discussion of results on bulk thermodynamics obtained with $\mathscr{O}\left(a^{2}\right)$ improved gauge and fermion actions.

\subsection{QCD Equation of State at vanishing chemical potential}

\subsubsection{Bulk thermodynamics: Trace anomaly and entropy density}

Lattice calculations of the QCD equation of state with almost physical light quark masses and a physical value of the strange quark mass have, so far, been performed only on lattices with temporal extent $N_{\tau}=4$ and 6 . These calculations have been performed on lines of constant physics (LCP), i.e. with bare quark masses chosen such that a set of hadron masses stays constant as the continuum limit is approached. In order to study QCD thermodynamics over a wide temperature range it is necessary to determine the parameters that characterize the LCP at zero temperature over a wide range of lattice cut-offs. On the LCP one, furthermore, has to calculate an additional observable that can be used to set the temperature scale for the finite-T calculations. As this scale has to be determined at zero temperature, i.e. on large lattices, for quite a few different cut-off values $(a)$ corresponding to the temperature values $\left(T=1 / N_{\tau} a\right)$ one wants to analyze, it is advantageous to use a gluonic observable, e.g. the static quark potential, that is easy to calculate, is not influenced strongly by the chiral sector of QCD and has a weak cut-off dependence. In this way it becomes possible to minimize the influence of spurious zero temperature cut-off effects on thermodynamic studies and to control the genuine cut-off and quark mass dependence of numerical calculations at finite temperature.

In Fig. 3 we show results for the trace anomaly, $\Theta^{\mu \mu}(T) / T^{4}=(\varepsilon-3 p) / T^{4}$, and the entropy density, $s / T^{3}=(\varepsilon+p) / T^{3}$, calculated with $\mathscr{O}\left(a^{2}\right)$ improved gauge and staggered fermion actions [5], 6]. We note that the entropy density as well as energy density and pressure are not independent observables but are obtained from $\Theta^{\mu \mu}(T) / T^{4}$ using standard thermodynamic relations, i.e. after having evaluated the trace anomaly on the lattice, one obtains the pressure through integration over the trace anomaly,

$$
\frac{p}{T^{4}}-\frac{p}{T_{0}^{4}}=\int_{T_{0}}^{T} \mathrm{~d} T^{\prime} \frac{\varepsilon-3 p}{T^{\prime 5}} .
$$

\footnotetext{
${ }^{1}$ For details on staggered fermion actions used in large scale studies of QCD thermodynamics we refer to the

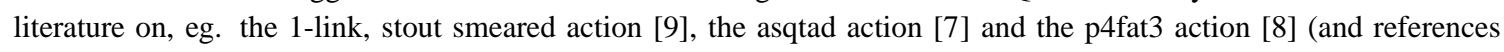
therein).

${ }^{2}$ It sometimes is attempted to correct for these cut-off effects by normalizing numerical results obtained at some value of the temperature in simulations on lattices with given temporal extent $N_{\tau}$ to the Stefan-Boltzmann value for pressure and energy density on the same size lattices at infinite temperature. This, however, does not solve the problem as cut-off effects are known to be temperature dependent. Moreover, it leads to thermodynamic inconsistencies when calculating, for instance, the trace anomaly, $(\varepsilon-3 p) / T^{4}$, which vanishes in the infinite temperature, ideal gas limit.
} 

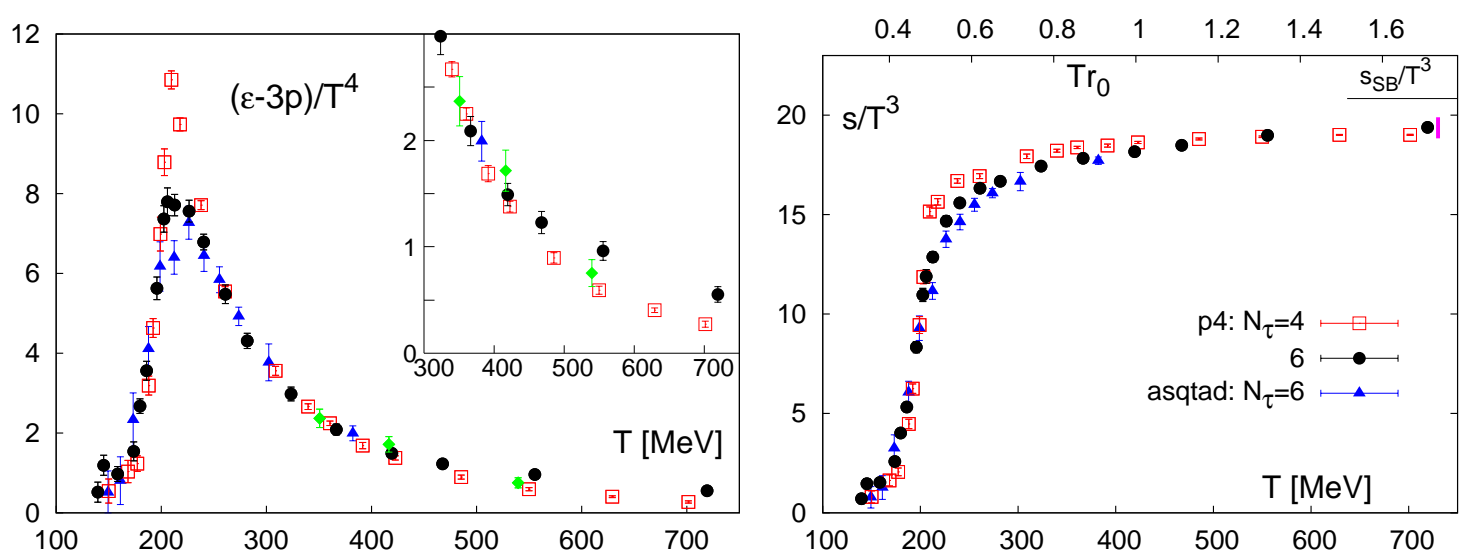

Figure 3: The trace anomaly, $\Theta^{\mu \mu}(T) \equiv \varepsilon-3 p$, in units of $T^{4}$ (left) and the entropy density, $s \equiv \varepsilon+p$, in units of $T^{3}$ calculated with the p4fat 3 action [6] on lattices with temporal extent $N_{\tau}=4$ and 6 . For $\Theta^{\mu \mu} / T^{4}$ we also show results on $N_{\tau}=8$ lattices (diamonds) obtained at high temperature. For $N_{\tau}=6$ results from calculations with the asqtad action are also shown [5]. In the right hand figure we also show the temperature scale $\operatorname{Tr}_{0}$ (upper X-axis) which has been obtained from an analysis of static quark potentials at zero temperature [6]. The MeV-scale shown on the lower $\mathrm{x}$-axis has been extracted from this using $r_{0}=0.469 \mathrm{fm}$.

Choosing the initial temperature $T_{0}$ deep enough in the hadronic phase where the pressure becomes exponentially small such that the contribution of $p\left(T_{0}\right) / T_{0}^{4}$ can be ignored safely, one obtains the energy and entropy densities through suitable combinations of $\Theta^{\mu \mu}(T)$ and $p(T)$. The small vertical bar in the right hand part of Fig. B(right) shows an estimate of the uncertainty that arises from setting the pressure to zero at $T_{0}=100 \mathrm{MeV}$. It is based on an estimate of the entropy density in a hadron resonance gas at this temperature [6].

In Fig. 3(left) we show results for the trace anomaly obtained in calculations with the p4fat 3 action on lattices with temporal extent $N_{\tau}=4,6$ and 8 on large spatial volumes, i.e. $V^{1 / 3} T=$ $N_{\sigma} / N_{\tau}=4$ or larger. The insertion in this figure shows the high temperature part of $(\varepsilon-3 p) / T^{4}$. Obviously cut-off effects are an issue in this regime. For $T \gtrsim 400 \mathrm{MeV}$, i.e. at temperatures larger than about twice the transition temperature, results on the $N_{\tau}=4$ lattice drop more rapidly than on the $N_{\tau}=6$ lattice. The calculations on the $N_{\tau}=8$ lattice, however, confirm the latter results and suggest that cut-off effects are under control in this high temperature region already on the $N_{\tau}=6$ lattices. This is consistent with the analysis of cut-off effects for the p4-action in the infinite temperature, ideal gas limit [14].

Also at $T \simeq 200 \mathrm{MeV}$, i.e. at temperatures just above the transition region, the trace anomaly shows some cut-off dependence. The peak in $(\varepsilon-3 p) / T^{4}$ is much more pronounced in the $N_{\tau}=4$ calculations than in the $N_{\tau}=6$ case. This can be traced back to the rapid change of non-perturbative $\beta$-functions in the crossover region from weak to strong coupling [6]. As can be seen from the entropy density shown in Fig. B(right) this leads to a noticeable cut-off dependence in $s / T^{3}$ in this temperature range. It, however, has little effect on the high temperature behavior. This also holds true for energy density and pressure [6]. It also is reassuring that calculations on performed $N_{\tau}=6$ lattices with the p4fat 3 action on quite large lattices, $V^{1 / 3} T=4$, are in good agreement 


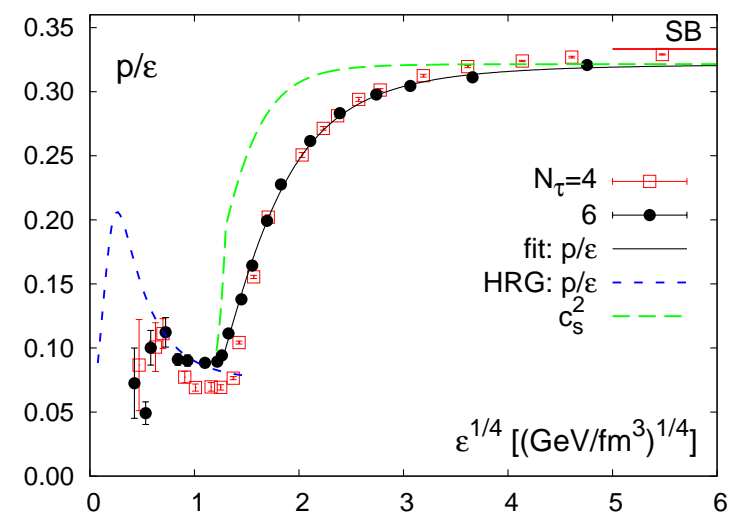

Figure 4: The ratio $p / \varepsilon$ as function of the fourth root of the energy density obtained from calculations with the p4fat 3 action on lattices with temporal extent $N_{\tau}=4$ and 6 . Also shown is the velocity of sound extracted from a fit to $p / \varepsilon[6]$ and using Eq. 2.2. The dashed curve at low energies shows the results for $p / \varepsilon$ calculated in a hadron resonance gas model (HRG).

with simulations that have been performed with the asqtad action on a smaller physical volume, $V^{1 / 3} T=2$ [5]. Results from the latter calculation are also shown in Fig. 3. The asqtad action has quite a different cut-off dependence at high temperature, it uses non-perturbatively improved (tadpole) couplings and also incorporates a more sophisticated smearing of 1-link terms in the staggered action to reduce flavor symmetry breaking effects. The good agreement between asqtad and p4fat 3 simulations thus suggests that these features only play a minor role in the common temperature range explored in both calculations, $150 \mathrm{MeV} \lesssim T \lesssim 400 \mathrm{MeV}$.

The results shown in Fig. 目 have been obtained in calculations with a physical strange quark mass and light quark masses that are about (2-2.5) times larger than in nature. This difference is of no significance at high temperature as the quark masses are small in units of the temperature ${ }^{3}$. It may, however, play a role in the low temperature hadronic phase. From the experience gained in simulations with different light quark masses [7, 8] it is to expected that the region of sudden rise in the trace anomaly as well as the entropy density shifts to somewhat smaller temperatures in the case of physical quark mass values. Cut-off effects will lead to a similar effect. This deserves a further careful analysis (see also disccusion in part II [11]).

\subsubsection{Equation of state and velocity of sound}

For the description of the expansion of dense matter created in heavy ion collisions, in particular its hydrodynamic modeling, the temperature dependence of bulk thermodynamic observables is not of direct interest. It is more relevant to get good control over the dependence of the pressure on the energy density, $p(\varepsilon)$, and deduce from this the velocity of sound,

$$
c_{s}^{2}=\frac{\mathrm{d} p}{\mathrm{~d} \varepsilon}=\varepsilon \frac{\mathrm{d} p / \varepsilon}{\mathrm{d} \varepsilon}+\frac{p}{\varepsilon} .
$$

\footnotetext{
${ }^{3}$ The renormalization group invariant light quark mass for the calculations performed with the p4fat 3 action has been estimated to be $m^{R G I}=8.0(4) \mathrm{MeV}[$ [
} 

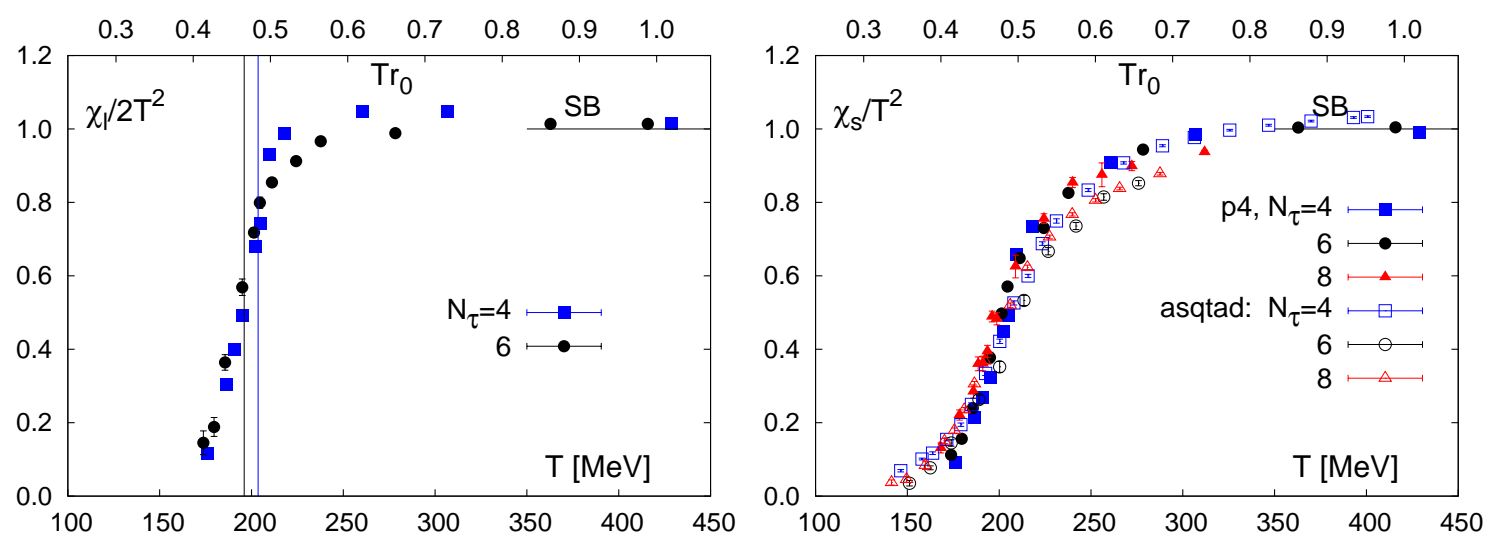

Figure 5: Light $\left(\chi_{l}\right)$ and strange $\left(\chi_{s}\right)$ quark number susceptibilities at vanishing chemical potential: The left hand side shows $\chi_{l} / 2 T^{2}$ calculated with the $\mathrm{p} 4 \mathrm{fat} 3$ action on lattices with temporal extent $N_{\tau}=4$ and 6 . Vertical lines indicate here the location of transition temperatures deduced from peaks in chiral susceptibilities [6] on lattices of same size. The right hand side shows $\chi_{s} / T^{2}$ calculated with p4fat 3 and asqtad actions on lattices with temporal extent $N_{\tau}=4,6[16]$ and 8 . All results are from calculations with a physical strange quark mass and almost physical, degenerate light quark masses, $m_{l}=0.1 m_{s}$. Data for $N_{\tau}=8$ are preliminary data of the hotQCD collaboration [17].

This is shown in Fig. \#. We note that the velocity of sound gets close to the ideal gas value at energy densities of about $15 \mathrm{GeV} / \mathrm{fm}^{3}$ or temperatures $T \simeq 300 \mathrm{MeV}$. However, for smaller energy densities, i.e. in the entire energy range of interest to the current RHIC experiments equation of state, $p(\varepsilon)$, as well as the velocity of sound drop rapidly. In the transition region, i.e. at $\varepsilon \simeq$ $1 \mathrm{GeV} / \mathrm{fm}^{3}$, one finds $c_{s}^{2} \simeq p / \varepsilon \simeq 0.09$.

We thus conclude that in the entire density regime, relevant for the expansion of dense matter created at RHIC, the QCD EoS shows large deviations from the conformal limit, $c_{s}^{2}=p / \varepsilon=1 / 3$.

\subsection{Non-vanishing chemical potential}

At small values of the quark chemical potential corrections to the equation of state can be evaluated systematically in terms of a Taylor expansion [15] in light $\left(\mu_{l}=\mu_{u}=\mu_{d}\right)$ and strange $\left(\mu_{s}\right)$ quark chemical potentials. The expansion coefficients are evaluated at vanishing values for the chemical potential and are related to thermal fluctuations of light quark and strangeness numbers in a thermal medium at $\mu_{l, s}=0$. The leading order, quadratic corrections are given in terms of the diagonal quark number susceptibilities $\chi_{l}$ and $\chi_{s}$ as well as an off-diagonal susceptibility $\chi_{l s}$,

$$
\frac{p\left(T, \mu_{l}, \mu_{s}\right)}{T^{4}}=\frac{p(T, 0,0)}{T^{4}}+\frac{1}{2} \frac{\chi_{l}}{T^{2}}\left(\frac{\mu_{l}}{T}\right)^{2}+\frac{1}{2} \frac{\chi_{s}}{T^{2}}\left(\frac{\mu_{s}}{T}\right)^{2}+\frac{\chi_{l s}}{T^{2}} \frac{\mu_{l}}{T} \frac{\mu_{s}}{T}+\mathscr{O}\left(\mu^{4}\right)
$$

with

$$
\frac{\chi_{q}}{T^{2}}=\frac{1}{V T^{3}} \frac{\partial^{2} \ln Z}{\partial\left(\mu_{q} / T\right)^{2}}, q=l, s, \frac{\chi_{l s}}{T^{2}}=\frac{1}{V T^{3}} \frac{\partial^{2} \ln Z}{\partial\left(\mu_{l} / T\right) \partial\left(\mu_{s} / T\right)} .
$$

In the infinite temperature limit these susceptibilities will approach in the continuum limit the value for massless two and one flavor quark number susceptibilities of an ideal quark gas, respectively, i.e. $\lim _{T \rightarrow \infty} \chi_{l} / T^{2}=2, \lim _{T \rightarrow \infty} \chi_{s} / T^{2}=1$ and $\lim _{T \rightarrow \infty} \chi_{l s} / T^{2}=0$. It is obvious from Fig. 国 that 
Taylor expansion coefficients of the pressure, eg. the quark number susceptibilities, suffer from similar cut-off effects as the pressure evaluated at $\mu_{l, s}=0$ and, on the other hand, profit also in the same way from a systematic $\mathscr{O}\left(a^{2}\right)$ improvement of the action.

Preliminary results for light and strange quark number susceptibilities, $\chi_{s, l}$, obtained with the p4fat 3 action on lattices with temporal extent $N_{\tau}=4$ and 6 [16] and the asqtad action [7] are shown in Fig. 5. The quark number susceptibilities change rapidly in the transition region. Like bulk thermodynamic observables, they are sensitive to deconfinement and reflect the rapid change from heavy hadronic degrees of freedom to light partonic degrees of freedom. In Fig. 5(right) we show in addition preliminary results for the strange quark number susceptibility obtained by the hotQCD collaboration from calculations on a lattice of temporal extent $N_{\tau}=8$ with the asqtad and p4fat 3 actions [17]. These results indicate a shift of the transition region to somewhat smaller temperatures, but otherwise follow the same pattern seen already in the calculations on the coarser $N_{\tau}=4,6$ lattices. We will discuss the deconfining features as well as the cut-off dependence seen in calculations of quark number susceptibilities in more detail in part II of this review. Here we only point out that $\chi_{l, s}$ have a temperature dependence very similar to that shown in Fig. 自 for the entropy density, which also is shared by energy density and pressure. For chemical potentials $\mu_{l} / T \leq 1$ they give the dominant finite- $\mu$ contribution to these thermodynamic observables, which stays below $10 \%$ for $\mu_{l} / T \lesssim 0.7$. This clearly is different for higher order derivatives of the partition function, that are sensitive to thermal fluctuations at non-zero baryon number density.

Quark number susceptibilities play an important role in the analysis of the QCD phase diagram at non-zero baryon chemical potential. If there exists a second order phase transition point at nonzero baryon chemical potential, the baryon (or light quark) number susceptibility will diverge at this point. It thus is important to get control over the dependence of $\chi_{l, s}$ on $\mu_{l}$. The leading order, quadratic corrections to $\chi_{l}$ and $\chi_{s}$ are given in terms of fourth order expansion coefficients of the pressure [18]; for vanishing $\mu_{s}$ one finds,

$$
\frac{\chi_{l}\left(\mu_{l}\right)}{T^{2}}=\frac{\chi_{l}(0)}{T^{2}}+12 c_{40}\left(\frac{\mu_{l}}{T}\right)^{2}+\mathscr{O}\left(\mu_{l}^{4}\right) \quad, \quad \frac{\chi_{s}\left(\mu_{l}\right)}{T^{2}}=\frac{\chi_{s}(0)}{T^{2}}+2 c_{22}\left(\frac{\mu_{l}}{T}\right)^{2}+\mathscr{O}\left(\mu_{l}^{4}\right),
$$

with expansion coefficients $c_{n m}=\left(V T^{3}\right)^{-1}\left[\left(\partial \ln \mathrm{Z}\left(T, \mu_{l}, \mu_{s}\right) / \partial\left(\mu_{l} / T\right)^{n} \partial\left(\mu_{s} / T\right)^{m}\right) / n ! / m !\right]_{\mu=0}$.

The expansion coefficients, $c_{40}$ and $c_{22}$, are positive and have a maximum at the temperature of the transition at vanishing $\mu_{l, s}$. For non-zero light quark chemical potential the susceptibilities thus increase and start developing a peak that becomes more pronounced with increasing $\mu_{l}$. This is obvious from Fig. 6 where we show baryon number and strangeness susceptibilities rather than light and strange quark number susceptibilities. The former are obtained in analogy to the latter after replacing in the QCD partition function the $(u, d, s)$ chemical potentials by appropriate linear combinations of $(B, Q, S)$ chemical potentials [16, 20, 21]. The leading order Taylor expansion for the baryon number susceptibility evaluated here as function of the baryon chemical potential, $\mu_{B}=2 \mu_{d}+\mu_{u} \equiv 3 \mu_{l}$, for vanishing strange quark chemical potential, $\mu_{S}=\mu_{d}-\mu_{s}$, and also for vanishing charge chemical potential, $\mu_{Q}=\mu_{u}-\mu_{d}$, is then given by ${ }^{4}$,

$$
\chi_{B}\left(\mu_{B}\right)=\frac{1}{9}\left(\chi_{l}\left(\mu_{B}\right)+\chi_{s}\left(\mu_{B}\right)+2 \chi_{l s}\left(\mu_{B}\right)\right) .
$$

${ }^{4}$ Other choices for the chemical potentials may be more suitable to resemble situations encountered, for instance, in heavy ion collision. Charge and strangeness chemical potentials may be adjusted to reproduce conditions met in a particular event-by-event analysis of fluctuations [19]. 

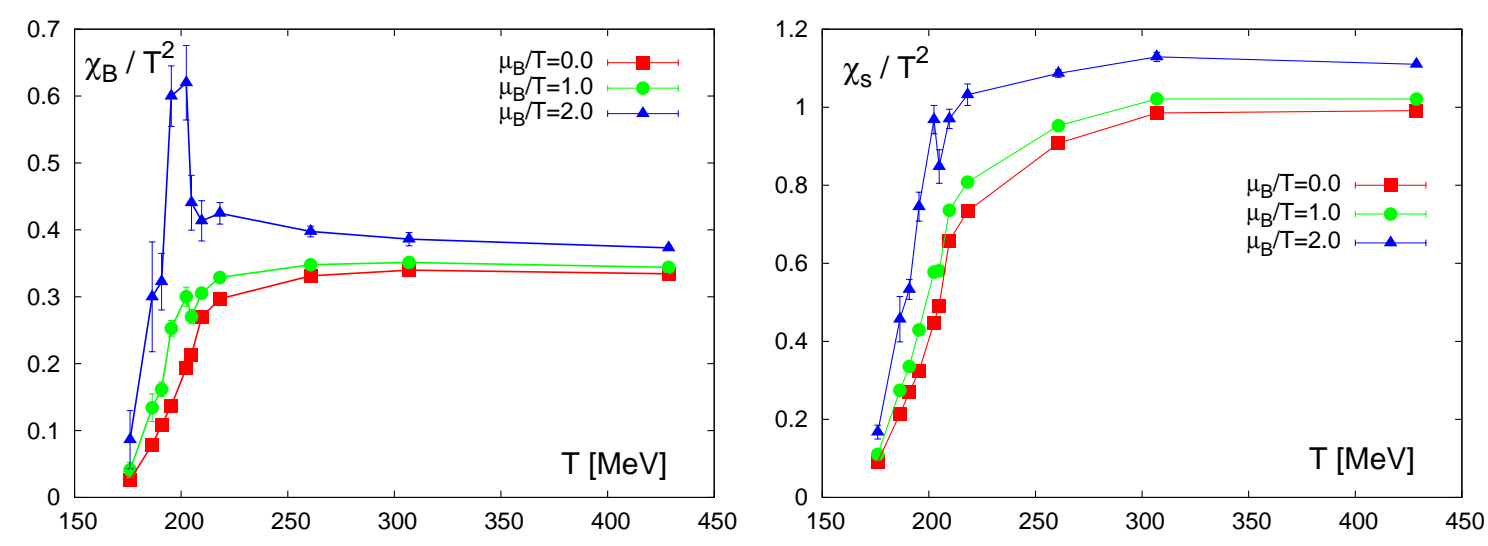

Figure 6: Fluctuations of baryon number (left) and strangeness (right) at non-zero baryon chemical potential calculated in leading order Taylor expansion on lattices with temporal extent $N_{\tau}=4$ in QCD with a physical strange quark mass and almost physical light quark masses, $m_{l}=0.1 m_{s}$.

We note that strangeness fluctuations do react to an increase in net baryon number $\left(\mu_{B}>0\right)$. This also holds true when the strange quark chemical potential is not chosen to be zero but is tuned such to insure that overall strangeness vanishes [16, 21]. The preliminary results shown in Fig. 6 so far have only been obtained on lattices with temporal extent $N_{\tau}=4$. Of course, one has to check the cut-off dependence also in this case through calculations on larger lattices [16]. Moreover, truncation effects in the Taylor expansion need to be analyzed by going beyond leading order in the expansion. In fact, higher order corrections are important! Only in the next order does the position of the peak become sensitive to the chemical potential and will get shifted towards lower temperatures with in creasing $\mu_{l}$.

\section{Conclusions}

Using $\mathscr{O}\left(a^{2}\right)$ improved staggered fermion actions with an almost physical quark mass spectrum much progress has been made in calculating the equation of state of QCD and extracting phenomenologically important quantities like the velocity of sound and fluctuations of hadronic charges. The current studies, performed mainly on lattices with temporal ex tent $N_{\tau}=4$ and 6 show some cut-off dependence. Calculations performed at high temperature for the trace anomaly on lattices with temporal extent $N_{\tau}=8$ suggest that these effects are small. A more detailed analysis, in particular at smaller temperatures in the hadronic phase, however, is still needed before firm conclusions on the EoS in the continuum limit can be drawn. Preliminary results obtained for quark number susceptibilities show that the transition region shifts to smaller temperatures with increasing $N_{\tau}$. This, of course, in accordance with the shift seen in systematic studies of the transition temperature performed with the p4fat $3[8]$ and asqtad [7]. actions. To quantify the effect for the equation of state will require a more detailed study on lattices with temporal extent $N_{\tau}=8$. 


\section{References}

[1] I. Arsene et al. [BRAHMS Collaboration], Nucl. Phys. A 757, 1 (2005);

B.B. Back et al. [PHOBOS Collaboration], Nucl. Phys. A 757, 28 (2005);

J. Adams et al. [STAR Collaboration], Nucl. Phys. A 757, 102 (2005);

K. Adcox et al. [PHENIX Collaboration], Nucl. Phys. A 757, 184 (2005).

[2] I. Horváth, A. D. Kennedy and S. Sint, Nucl. Phys. B 73, 834 (1999);

M. A. Clark, A. D. Kennedy and Z. Sroczynski, Nucl. Phys. Proc. Suppl. 140, 835 (2005).

[3] for an introductory survey see: F. Karsch, Lect. Notes Phys. 583, 209 (2002).

[4] Y. Aoki, Z. Fodor, S. D. Katz and K. K. Szabo, JHEP 0601, 089 (2006).

[5] C. Bernard et al., Phys. Rev. D 75, 094505 (2007).

[6] M. Cheng et al. [RBC-Bielefeld Collaboration], The QCD Equation of State with almost Physical Quark Masses, arXiv:0710.0354v1 [hep-lat]

[7] C. Bernard et al. [MILC Collaboration], Phys. Rev. D 71, 034504 (2005).

[8] M. Cheng et al. [RBC-Bielefeld Collaboration], Phys. Rev. D 74, 054507 (2006).

[9] Y. Aoki, Z. Fodor, S. D. Katz and K. K. Szabo, Phys. Lett. B 643, 46 (2006).

[10] for a recent review see, Ch. Schmidt, PoS LAT2006, 021 (2006).

[11] F. Karsch, Recent lattice results on finite temperature and density QCD, part II, PoS (Lattice 2007) 015.

[12] C.W. Bernard et al. [MILC Collaboration], Phys. Rev. D 55, 6861 (1997).

[13] A. Ali Khan et al., Phys. Rev. D 65, 074510 (2001).

[14] U. M. Heller, F. Karsch and B. Sturm, Phys. Rev. D 60, 114502 (1999).

[15] R.V. Gavai and S. Gupta, Phys. Rev. D 68034506 (2003).

[16] C. Miao and C. Schmidt (for the RBC-Bielefeld Collaboration), Bulk thermodynamics and charge fluctuations at non-vanishing baryon density, PoS (LATTICE2007) 175.

[17] C. DeTar and R. Gupta (for the hotQCD Collaboration), Toward a precise determination of $T_{c}$ with 2+1 flavors of quarks, PoS (LATTICE2007) 179.

[18] C. R. Allton et al., Phys. Rev. D 71, 054508 (2005).

[19] V.V. Begun et al., Phys. Rev. C 76, 024902 (2007).

[20] R. V. Gavai and S. Gupta, Phys. Rev. D 73, 014004 (2006).

[21] C. Bernard et al. [MILC Collaboration], The equation of state with nonzero chemical potential for 2+1 flavors, PoS (LATTICE2007) 190. 